\title{
Ballistic Hole Emission Microscopy on Metal-Semiconductor Interfaces
}

\author{
T. Banerjee, E. Haq, M. H. Siekman, J. C. Lodder, and R. Jansen \\ $\mathrm{MESA}^{+}$Institute for Nanotechnology, University of Twente, 7500 AE Enschede, The Netherlands
}

\begin{abstract}
The transport of hot holes across metal-semiconductor interfaces is studied using ballistic hole emission microscopy. From the tip of a scanning tunneling microscope nonequilibrium holes are injected into a thin metallic overlayer on a p-type Si semiconductor, inducing a current of holes into the Si valence band. We have studied hole transport across interfaces between p-type Si and different metals (Au, $\mathrm{Cu}$, and $\mathrm{Al}$ ). It is found that the magnitude of the transmitted hole current depends strongly on the type of metal, the Schottky barrier height, and the energy distribution of the holes. In addition, we show that a significant yet smaller hole current can be induced in the reverse case where the tip is used to inject hot electrons, generating holes during inelastic decay in the metal overlayer. The results are compared to recent results on spin-dependent hole transmission in ferromagnet/p-type semiconductor structures.
\end{abstract}

Index Terms-Hot carriers, microscopy-ballistic hole emission, semiconductor-metal interfaces, spin dependent tunneling.

\section{INTRODUCTION}

B ALLISTIC electron emission microscopy (BEEM) [1] is widely used to study nanoscale properties of metal-semiconductor interfaces, ultrathin gate and tunnel oxides, and metallic and silicide thin films [2]-[4]. Its spin-sensitive counterpart, ballistic electron magnetic microscopy (BEMM) [5], can be used to probe buried interfaces in semiconductor-ferromagnet hybrid structures, employing the spin-dependent transmission of hot electrons in ferromagnetic thin films. The technique was demonstrated to allow magnetic imaging with nanoscale resolution [6].

Recently, spin-dependent transmission of hot holes, rather than electrons, was demonstrated in a p-type semiconductor-ferromagnet hybrid structure [7] using ballistic hole emission microscopy (BHEM). This opened up a new route for fundamental studies of spin dynamics of nonequilibrium carriers in ferromagnets and also for realizing complementary spintronic devices. In BHEM [8], [9] nonequilibrium carriers emitted from the tip of a scanning tunneling microscope (STM) are injected locally into a (ferromagnetic) metal overlayer on a p-type semiconductor as is shown in Fig. 1(a). The tip bias polarity is positive, such that a current of hot holes is injected by tunneling. Depending on the scattering in the metallic layers, a fraction of the hot holes is transmitted into the valence band of the semiconductor, provided the energy and momentum criteria for crossing the metal-semiconductor Schottky barrier are satisfied. In another recent work, large spin-dependent effects were also observed in the reverse mode of ballistic hole emission microscopy (R-BHEM) [10]. As shown in Fig. 1(b), in this reverse mode the tip voltage is negative, such that hot electrons are injected into the metal overlayer. During transmission of the metal stack, inelastic decay via electron-hole (e-h) pair excitation creates holes that can be collected in the valence band of the underlying p-type semiconductor. With this technique, magnetic imaging with nanometer resolution has been demonstrated [10]. Interestingly, it was found that the hole current induced by hot-elec-

Digital Object Identifier 10.1109/TMAG.2005.854738 (a)

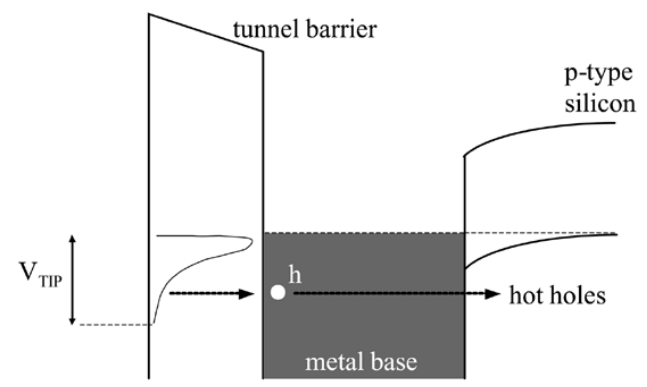

(b)

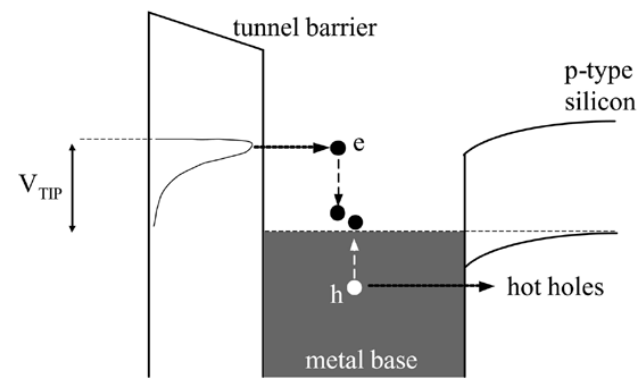

Fig. 1. (a) Schematic energy diagram of ballistic hole emission microscopy in direct mode (BHEM). A distribution of holes is injected by tunneling from a STM tip into a metal film on a p-type Si semiconductor. The transmitted holes are collected in the Si valence band. (b) Schematic energy diagram of ballistic hole emission microscopy in reverse mode (R-BHEM). The STM tip at opposite bias polarity is used to inject hot electrons. Inelastic scattering of the hot electrons in the metal base creates electron-hole (e-h) pairs, the holes of which can be collected in the valence band of the semiconductor.

tron injection in reverse mode is significantly larger than that obtained for the case of direct hole injection.

In order to understand the spin-dependent transport of holes in these ferromagnet/semiconductor structures, it is important to examine the role of the Schottky barrier between the nonmagnetic metal and the p-type semiconductor collector. In this work we have studied the transport across metal/p-type $\mathrm{Si}(100)$ interfaces using various metals $(\mathrm{Au}, \mathrm{Cu}$, and $\mathrm{Al})$. We compare the transmission of holes for these different interfaces for direct BHEM as well as for reverse mode (R-BHEM). We find that the transmission is significantly larger for lower Schottky barrier interfaces as a result of the energy distribution of the hot 


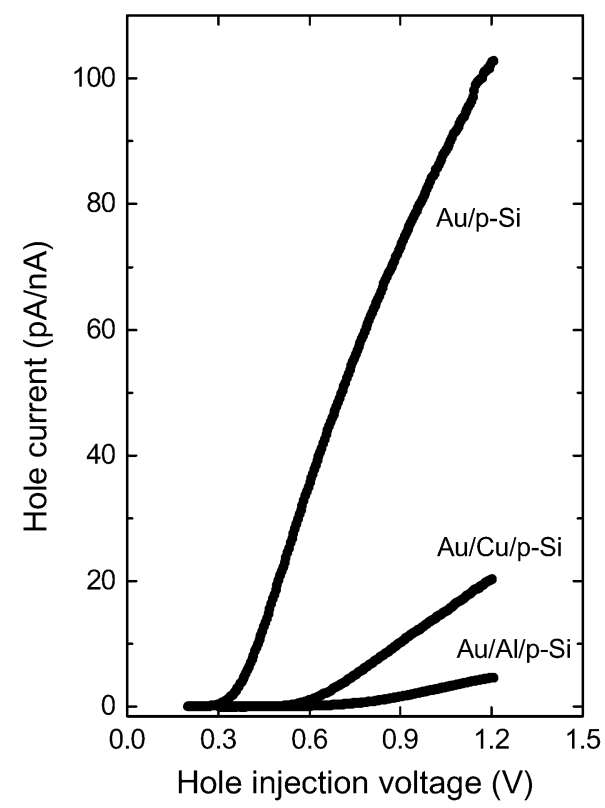

Fig. 2. Hole current per nA of injected holes versus positive tip voltage (BHEM mode) for $\mathrm{Au}(10 \mathrm{~nm}) / \mathrm{p}-\mathrm{Si}, \mathrm{Au}(3 \mathrm{~nm}) / \mathrm{Cu}(10 \mathrm{~nm}) / \mathrm{p}-\mathrm{Si}$, and $\mathrm{Au}(3 \mathrm{~nm}) /$ $\mathrm{Al}(10 \mathrm{~nm}) / \mathrm{p}-\mathrm{Si} . T=150 \mathrm{~K}$.

holes. We also show that the hole current for the R-BHEM is consistently lower than for the direct hole injection, opposite to the case where the overlayer contains ferromagnetic metals as in [7] and [10].

\section{EXPERIMENTAL DETAILS}

Samples used in this study are $\mathrm{Au}(30 \AA) / \mathrm{metal} / \mathrm{p}-\mathrm{Si}$, where the metal layer is either $70 \AA \mathrm{Au}$, or $100 \AA \mathrm{Cu}$ or Al. They were deposited by thermal evaporation in a molecular beam epitaxy system with a base pressure of $10^{-10}$ mbar. Substrates consist of HF-etched $\mathrm{p}-\mathrm{Si}(100)$ with a lithographically defined area of $150 \mu$ m diameter, surrounded by a thick $\mathrm{SiO}_{2}$ insulator. The $\mathrm{Au}$ cap layer of $30 \AA$ is deposited to provide a chemically inert surface for ex situ sample transfer to the ultrahigh vacuum STM system with a base pressure of $10^{-10}$ mbar. BHEM measurements are performed at $150 \mathrm{~K}$ using PtIr tips, with the metal overlayer of the sample grounded. A contact to the back of the p-type $\mathrm{Si}$ is used to detect the hole current $I_{\text {hole }}$ with a two-stage amplifier $\left(10^{11} \mathrm{~V} / \mathrm{A}\right)$ and a $300 \mathrm{~Hz}$ low-pass filter. Hole current spectra were recorded at several locations, and at least 15 spectra were averaged at each location. The spectra presented here therefore are representative of the whole surface. More details are described elsewhere [11]. The metal films are polycrystalline and have grain size and roughness comparable to that reported previously for structures grown on n-type $\mathrm{Si}$ [11].

\section{RESULTS AND DISCUSSION}

Representative hole spectra for $\mathrm{Au}(30 \AA) / \mathrm{metal} / \mathrm{p}$-Si structures are shown in Fig. 2, obtained by sweeping the tip bias while recording the hole current $\left(I_{\text {hole }}\right)$ at a constant injection current of $I_{T}=1 \mathrm{nA}$. The sign of $I_{\text {hole }}$ corresponds to holes flowing from the metal stack into the semiconductor back contact. For $\mathrm{Au} / \mathrm{p}-\mathrm{Si}$, a rather sharp onset of the transmitted hole current is

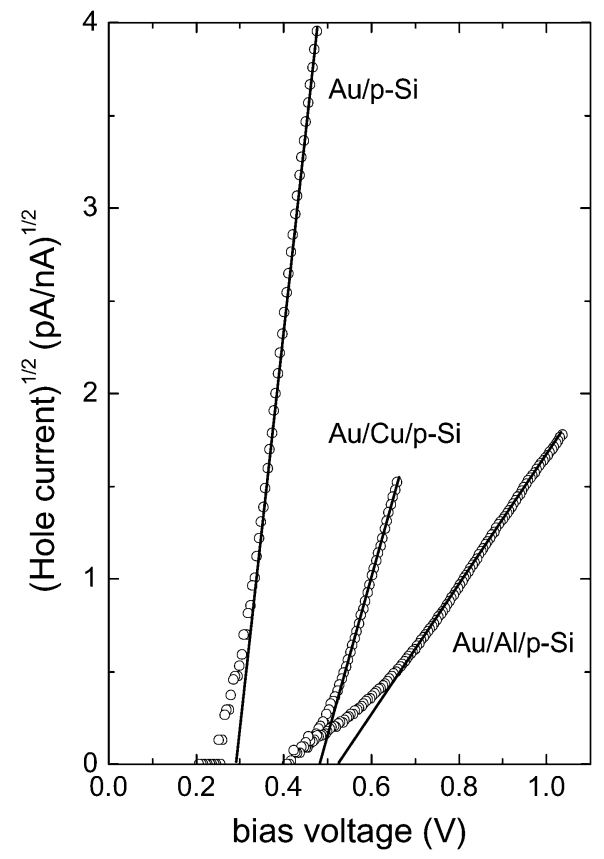

Fig. 3. Same data as in Fig. 2, but with the square root of the hole current plotted (symbols). The intercepts of the solid lines with the voltage axis correspond to the Schottky barrier heights.

observed around $0.3 \mathrm{eV}$, corresponding to the Schottky barrier height $\Phi_{b}$ of the $\mathrm{Au} / \mathrm{p}-\mathrm{Si}$ interface. For the case of $\mathrm{Au} / \mathrm{Cu} / \mathrm{p}-\mathrm{Si}$, the transmitted hole current is smaller by a factor of five as compared to Au. The onset of $I_{\text {hole }}$ occurs at a higher bias due to the different value of $\Phi_{b}$ for $\mathrm{Cu} / \mathrm{p}-\mathrm{Si}$. The hole transmission for the $\mathrm{Au} / \mathrm{Al} / \mathrm{p}-\mathrm{Si}$ structure is further reduced by a factor of five as compared to that of $\mathrm{Cu}$. The Schottky barrier heights can be extracted by plotting the square root of $I_{\text {hole }}$ as a function of the injection bias V (see Fig. 3), since near the threshold the current is expected to vary as $\left(V-\Phi_{b}\right)^{2}$. The intercepts of the solid lines with the voltage axis then correspond to the Schottky barrier height. For the $\mathrm{Au}, \mathrm{Cu}$, and $\mathrm{Al}$ we find $\Phi_{b}$ is $0.29 \pm 0.03 \mathrm{eV}$, $0.48 \pm 0.03 \mathrm{eV}$, and $0.52 \pm 0.03 \mathrm{eV}$, respectively.

The hole transmission of $10 \%$ at $+1.2 \mathrm{~V}$ for $\mathrm{Au}$ is rather large, despite the fact that the energy distribution of the holes injected by tunneling is peaked near the fermi level of the metal base film (see Fig. 1(a) and [8], [9]). Thus, a significant fraction of the injected holes do not have sufficient energy to overcome the Schottky barrier, even if no scattering occurs. The large $I_{\text {hole }}$ indicates highly ballistic transport of the holes into the zone-centered $\mathrm{Si}$ valence bands and suggests a long inelastic lifetime of hot holes in $\mathrm{Au}$, in agreement with recent calculations [12]. For $\mathrm{Cu}$, the calculated inelastic lifetime is long, [12] just as in $\mathrm{Au}$. Therefore, inelastic decay of the holes in the $\mathrm{Cu}$ film cannot explain the factor of five lower $I_{\text {hole }}$ for the sample with $\mathrm{Cu}$. We therefore attribute the difference to the value of $\Phi_{b}$, which for $\mathrm{Cu}$ is much larger than for Au. Given the energy distribution of the holes injected by tunneling, a smaller transmission is expected for higher $\Phi_{b}$ since the hole current arises mostly from the tail of the energy distribution. This accounts for most of the difference, although some scattering at the additional $\mathrm{Au} / \mathrm{Cu}$ interface may also contribute. However, we note that $\mathrm{Au} / \mathrm{Cu}$ interfaces are expected to be rather transparent since the bandstructure of Au and 


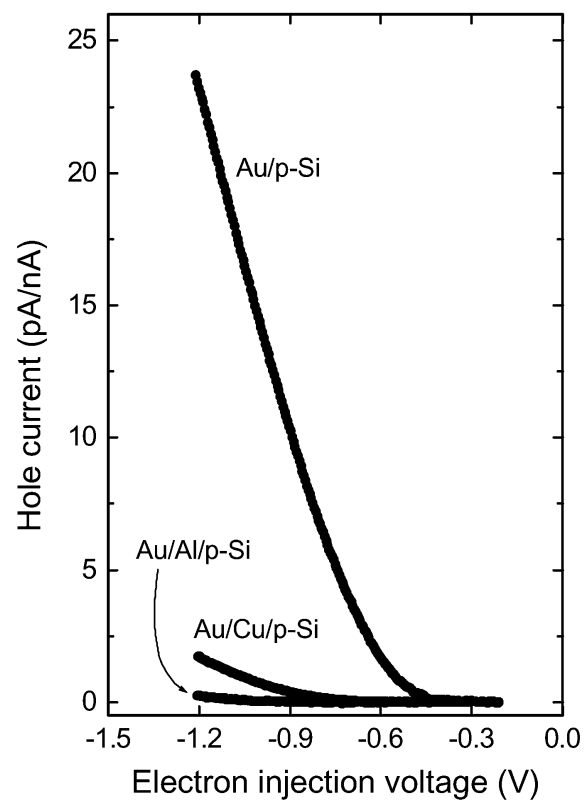

Fig. 4. Hole current per $\mathrm{nA}$ of injected electrons versus negative tip voltage (R-BHEM mode) for the same three samples as in Fig. 2. $T=150 \mathrm{~K}$.

TABLE I

Hole CURRENT For DiRECT AND REVERSE BHEM AND SCHOTTKY BARRIER HEIGHT $\Phi_{b}$ FOR DIFFERENT METAL/P-SILICON CONTACTS

\begin{tabular}{cccc}
\hline structure & $\begin{array}{c}\text { BHEM current } \\
\text { at } 1.2 \mathrm{~V}(\mathrm{pA} / \mathrm{nA})\end{array}$ & $\begin{array}{c}\text { R-BHEM current } \\
\text { at }-1.2 \mathrm{~V}(\mathrm{pA} / \mathrm{nA})\end{array}$ & $\begin{array}{c}\Phi_{b} \\
(\mathrm{eV})\end{array}$ \\
\hline $\mathrm{Au} / \mathrm{p}-\mathrm{Si}$ & 102 & 23 & 0.29 \\
\hline $\mathrm{Au} / \mathrm{Cu} / \mathrm{p}-\mathrm{Si}$ & 20 & 1.8 & 0.48 \\
\hline $\mathrm{Au} / \mathrm{Al} / \mathrm{p}-\mathrm{Si}$ & 4.3 & 0.2 & 0.52 \\
\hline
\end{tabular}

$\mathrm{Cu}$ is quite similar in the relevant energy range. For the structure with $\mathrm{Al}$, the Schottky barrier is only slightly higher than for sample with $\mathrm{Cu}$. This, therefore, cannot account for the much lower hole current for the $\mathrm{Al}$ sample. Since the bandstructure of $\mathrm{Au}$ and $\mathrm{Al}$ are quite different, the hole transmision of a $\mathrm{Au} / \mathrm{Al}$ interface is expected to be smaller than that of a $\mathrm{Au} / \mathrm{Cu}$ interface. Moreover, during transport of the $\mathrm{Al}$ film the hole current is expected to be more strongly attenuated, since the calculated lifetimes of hot holes in $\mathrm{Al}$ are smaller than in noble metals such as $\mathrm{Au}$ and $\mathrm{Cu}$ [13].

In Fig. 4, we show spectra for the same structures as in Fig. 2, but obtained in reverse mode (R-BHEM) using negative tip bias, producing injection of hot electrons. The sign of the collected current is the same as for the direct BHEM case, proving the collection of holes. We again observe that $I_{\text {hole }}$ is largest for the $\mathrm{Au}$ film, and smallest for the $\mathrm{Al} \mathrm{film}$. However, the reduction in current when going from $\mathrm{Au}$ to $\mathrm{Cu}$ to $\mathrm{Al}$ is more strong, about a factor of 10 in each step, as can be seen in Table I where the transmission results for direct BHEM and reverse BHEM are summarized.

Comparing the hole current for direct BHEM and reverse BHEM, we find that the latter is smaller for all the structures studied. Two factors are responsible. The hot holes created by the inelastic decay of the injected hot electrons have a more isotropic momentum distribution than holes injected by tunneling, which produces a more forward focused momentum dis- tribution. In addition, the energy distribution of the holes created by the inelastic decay of electrons via electron-hole $(\mathrm{e}-\mathrm{h})$ pair excitation is different from the energy distribution of holes injected by tunneling. A similar trend was also observed in [8], [9] for Au on p-type Si. This is opposite to the case where the overlayer contains ferromagnetic metals as in [7] and [10], where a larger hole current was observed in the R-BHEM case.

\section{CONCLUSION}

The transport of hot holes across metal/p-Si(100) interfaces has been studied using ballistic hole emission microscopy for both direct and reverse mode. We find that the magnitude of the collected hole current depends strongly on the type of metal, the Schottky barrier height, and the energy distribution of the holes, with consistently smaller hole current for the reverse mode.

\section{ACKNOWLEDGMENT}

This work was supported by the Dutch Foundation for Fundamental Research on Matter (FOM), the Royal Netherlands Academy of Arts and Sciences (KNAW), and the Dutch Technology Foundation (STW).

\section{REFERENCES}

[1] W. J. Kaiser and L. D. Bell, "Direct investigation of subsurface interface electronic structure by ballistic-electron-emission microscopy," Phys. Rev. Lett., vol. 60, no. 14, pp. 1406-1409, 1988.

[2] M. Prietsch, "Ballistic-electron emission microscopy (BEEM): Studies of metal/semiconductor interfaces with nanometer resolution," Phys. Rep., vol. 253, pp. 163-233, 1995.

[3] R. Ludeke, A. Bauer, and E. Cartier, "Hot electron transport in $\mathrm{SiO}_{2}$ probed with a scanning tunnel microscope," Appl. Phys. Lett., vol. 66, no. 6, pp. 730-732, 1995.

[4] T. Meyer and H. von Känel, "Study of interfacial point defects by ballistic electron emission microscopy," Phys. Rev. Lett., vol. 78, no. 16, pp. 3133-3136, 1997.

[5] W. H. Rippard, A. C. Perrella, and R. A. Buhrman, "Ballistic electron microscopy study of ultrathin oxidized aluminum barriers for magnetic tunnel junctions," Appl. Phys. Lett., vol. 78, no. 11, pp. 1601-1603, 2001.

[6] W. H. Rippard and R. A. Buhrman, "Ballistic electron magnetic microscopy: Imaging magnetic domains with nanometer resolution," Appl. Phys. Lett., vol. 75, no. 7, pp. 1001-1003, 1999.

[7] T. Banerjee, E. Haq, M. H. Siekman, J. C. Lodder, and R. Jansen, "Spin filtering of hot holes in a metallic ferromagnet," Phys. Rev. Lett., vol. 94, p. $027204,2005$.

[8] M. H. Hecht, L. D. Bell, W. J. Kaiser, and L. C. Davis, "Ballistic-hole spectroscopy of interfaces," Phys. Rev. B, vol. 42, no. 12, pp. 7663-7666, 1990.

[9] L. D. Bell, M. H. Hecht, W. J. Kaiser, and L. C. Davis, "Direct spectroscopy of electron and hole scattering," Phys. Rev. Lett., vol. 64, no. 22, pp. 2679-2682, 1990.

[10] E. Haq, T. Banerjee, M. H. Siekman, J. C. Lodder, and R. Jansen, "Ballistic hole magnetic microscopy," Appl. Phys. Lett., vol. 86, no. 8, p. $082502,2005$.

[11] E. Haq et al., "Nanoscale magnetic hysteresis of $\mathrm{Ni}_{80} \mathrm{Fe}_{20} / \mathrm{Au} / \mathrm{Co}$ trilayers using ballistic electron magnetic microscopy," J. Appl. Phys., vol. 95, no. 11, pp. 6930-6932, 2004.

[12] I. Campillo, A. Rubio, J. M. Pitarke, A. Goldmann, and P. M. Echenique, "Hole dynamics in noble metals," Phys. Rev. Lett., vol. 85, no. 15, pp. 3241-3244, 2000.

[13] V. P. Zhukov and E. V. Chulkov, "Lifetimes of low-energy electron excitations in metals," J. Phys.: Condens. Matter, vol. 14, pp. 1937-1947, 2002.

Manuscript received February 7, 2005 